\title{
Improving Customer Retention Through Service Quality At Call Centers
}

Elaine J. Labach, Troy University, USA

\begin{abstract}
For many call centers, improving customer service is an ongoing goal. These inbound call service centers have a unique opportunity to impact customer retention through value-adding customer service interactions. This paper serves three objectives: validate the connection between service quality, customer service and retention; describe a quality process for improving service quality; and discuss training and other key implementation needs for improving service delivery.
\end{abstract}

Keywords: Call Center, Customer Retention, Service Quality

\section{CUSTOMER SATISFACTION AND RETENTION}

$\mathcal{R}$ ecent studies show that customers are becoming more dissatisfied with call centers, and inbound call centers are "on trial" with every interaction from customers they serve (Jaiswal, 2008, p. 406). Call centers are challenged with balancing efficiency with ever-increasing customer satisfaction requirements and many call centers struggle with achieving both simultaneously. Philip Crosby's definition of quality can be used to define service quality as conformance to specifications (Crosby, 1979). Crosby stated that any product or service that consistently replicated its design specifications would have high quality. Crosby went on to add that if quality is continuously improved, total costs would fall, allowing a company to increase its profitability. This reasoning led to Crosby's most famous claim - that quality was "free". By linking improved service delivery to higher levels of customer satisfaction and retention, call centers can improve their competitiveness. They can lower their total costs by keeping a higher percentage of current customers vs. incurring the additional costs of acquiring replacement customers. By improving service delivery, or contact quality, call centers can increase customer retention and add to their bottom line. Key aspects of improved service quality are delivering fast initial response and prompt, or one call, resolution. (Call centers are also looking toward the internet to help them improve the quality of the customer interaction with increased functionality and greater choices.)

From a service delivery perspective, the service profit chain can be used to show the linkages between external service value as perceived by the customer, customer loyalty (i.e., retention) and profitability (as highlighted in the Marketing/Sales block in Figure 1). The service profit chain is described in Figure 1 as taken from Heskett, J., Jones, T., Loveman, G., Sasser Jr., E., \& Schlesinger, L. (1994) and discussed in Labach (2009).

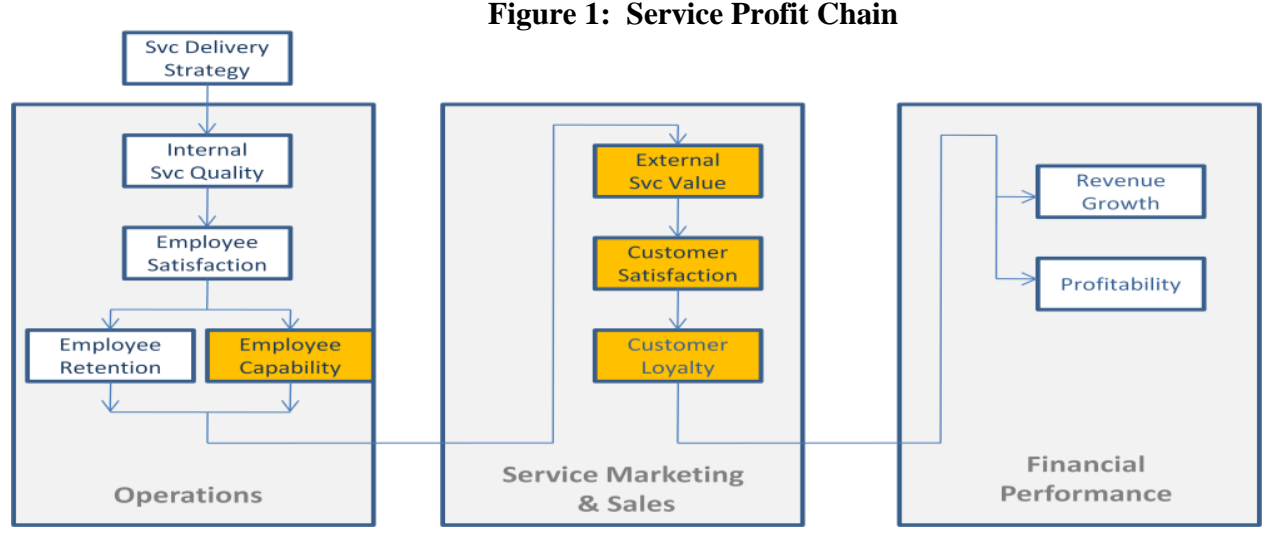


One of the linkages predicted by the service profit chain is that between customer satisfaction and loyalty (i.e., retention). Although industry call center customer retention statistics are not available, it is the author's opinion that many retention curves look like the one shown in Figure 2 (based on the US automotive insurance industry). Over time, the number of customers a business keeps decreases, especially during the early stages of the relationship. It is not uncommon for insurance service organizations to lose half of their new customers by the end of the first year, and of those that remain, another half may leave by the end of the second year. Therefore, the biggest opportunity for call centers to improve customer retention is in the early stages of interacting with their new customers (within the first year). Given the inherent characteristics of inbound call centers, service delivery can positively impact customer satisfaction. And, customer satisfaction is one way to positively impact customer retention, although it is recognized as only one aspect that drives customer satisfaction. Business processes and procedures, along with the quality of the product, also play key roles in retaining customers.

Figure 2: General Customer Retention Curve

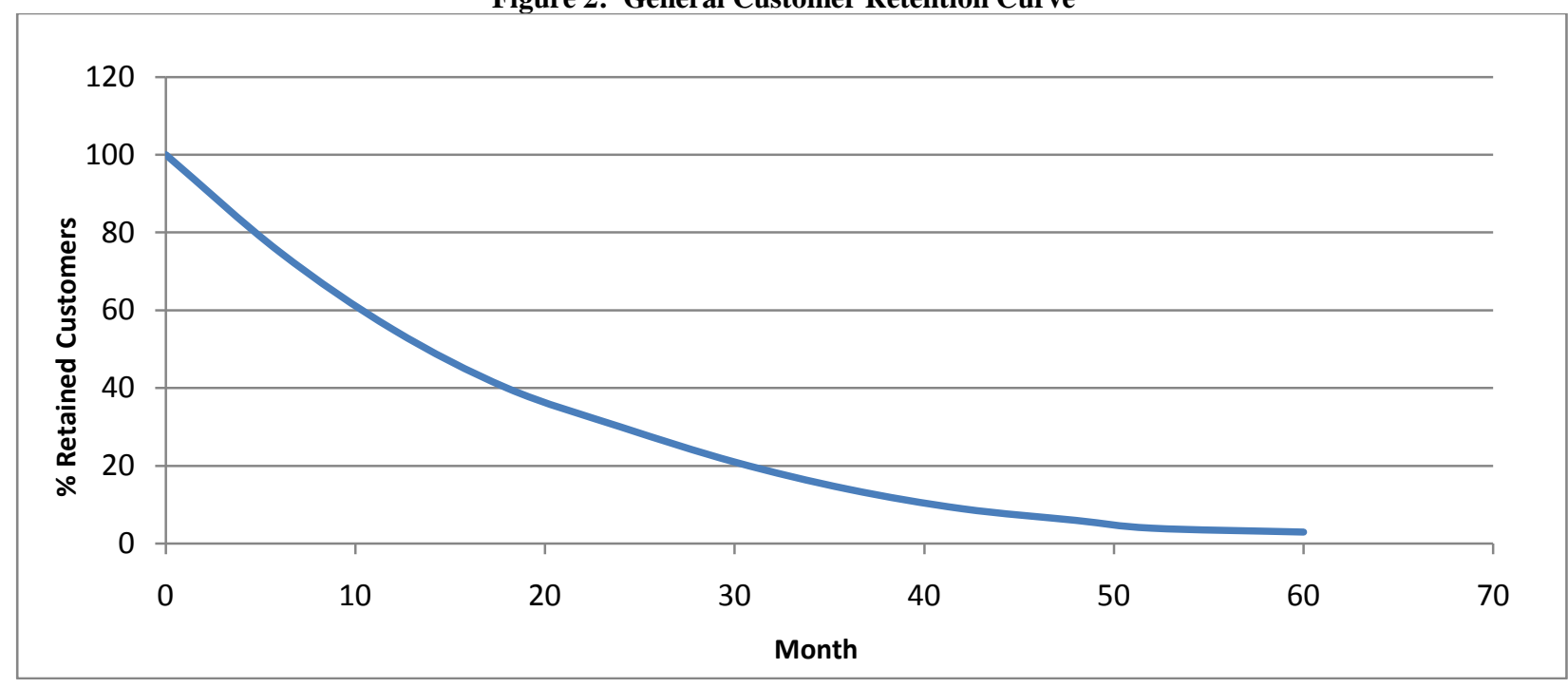

From a profitability standpoint, Heskett et al (1994) estimate that a 5\% increase in customer loyalty can produce profit increases from $25-85 \%$. Based on the author's professional experience in the insurance industry, is not uncommon that a $1 \%$ increase in customer retention amounts to $\$ 20-\$ 50$ million in revenues. So, the profitability impact is a big motivation for call center organizations to focus on improving customer retention.

And, it can be expected that the retention rate for very satisfied customers is higher than those who rate their experience as satisfied. Identifying this percentage provides an additional incentive for improving many satisfied customers to very satisfied ones.

\section{SERVICE DELIVERY AND CUSTOMER SATISFACTION}

Customer satisfaction and service quality are two distinct, but related constructs (Bansal \& Taylor, 1997; Dabholkar, Shepherd \& Thorpe, 2000). Dabholkar et al (2000) recommend that although related, customer satisfaction should be measured separately from service quality in order to understand how customers evaluate service quality. Many US call centers secure third-parties to measure customer satisfaction, either real time right after the call or with a survey at the end of an online transaction (via email or chat). Customer satisfaction is most often measured on a 5-point scale, with very satisfied scoring a level of 5. This rating is often called "top box" satisfaction and indicates the highest level of satisfaction that can be achieved (Jaiswal, 2008, p. 407). Jaiswal (2008) indicates that another way of reporting customer satisfaction is through "top two" and "bottom two" averages (p. 407). The top two average indicates the percentage of customers who scored service at a 4 (satisfied) or 5 (very satisfied) level. The bottom two average indicates the percentage of customers who scored service at a 1 (very 
dissatisfied) or 2 (dissatisfied) level. There are no public industry statistics available on what the current percentage of very satisfied calls reported for the various call center industries. Jaiswal (2008) reports that a captive call center reported $65 \%$ of calls with at least a four rating (satisfied) (p. 406 ). Based on the author's experience, US inbound call centers that are focusing on improving service may see at least $50 \%$ of their calls rated a 5 with $70-80 \%$ of calls scoring in top two scores. Upon reviewing these customer satisfaction results, there are two improvement goals. The first is elevating as many of the satisfied customers (score of 4) to very satisfied (score of 5). The second goal is to reduce the percentage of bottom two averages and elevate the satisfaction scores of these types of calls. One of the areas where improvement has yielded a positive increase in low customer satisfaction scores has been in implementing first call resolution. Although there is some variation in how first call resolution is defined (either an additional call to the customer is not required or no additional paperwork is required to be finished after the call), Levin (2007) reports that for every one percent improvement in first call resolution, customer satisfaction improved by one percent (p. 24).

\section{Service Quality Improvement}

Service quality is typically measured internally at call centers, where calls are monitored either real time or after they occur. Many call centers have established dimensions of a call that they deem as exceptional in service quality, and they typically compare a sample of calls to either an overall internal goal (i.e., $80 \%$ of calls should be what we would rate internally as a 5) or to a list of criteria that relate to a benchmarked list of dimensions. These internal approaches are necessary for two reasons, with the first reason relating to how customer satisfaction feedback is provided. Customer satisfaction levels are often provided in aggregate (by call center site vs. by call). Reports will have top box results for a given time period but do not allow insights into how to improve the scores. Also, these results are rarely provided real time, and it is common for a call center to see its results after a significant lapse in time has occurred. The second reason for internal measuring processes is because of gaps in customer perception in evaluating the call. Because customers have had a wide variety of call center experiences where they have received less than exceptional service from other providers, customers often have lower expectations than the goal set by many call centers. Therefore, there is often a gap between the goal a call center will set and the results received from customer satisfaction surveys ( although this service level gap is decreasing). Whether customer satisfaction results or internal benchmark goals are used, the focus needs to be on continuously improving how the service is delivered, and not solely on top box customer satisfaction performance measures. And whatever the goal, improved customer satisfaction will ultimately result in increases in customer retention, which will drive improved financial performance.

\section{Using Feedback Rules to Assess Calls}

From a quality perspective, six feedback rules should be considered in setting up an improvement process to assess calls as shown in Figure 2.

Figure 2: Six Feedback Rules in Assessing Calls

Feedback should be:

1. focused on important indicator

2. accurate

3. timely

4. given to those who can improve the process

5. focused on behaviors and/or skills that can be changed or improved

6. Management "owns" the feedback process

The first feedback rule is that the assessment should be focused and specific to an area that directly impacts a KSI (key success indicator) - at many call centers, service delivery is an important KSI. 
The second and third feedback rules concern the accuracy and timeliness of the feedback on service delivery. From an accuracy perspective, the feedback should not be biased. Bias can occur when managers evaluate the calls of their own CSRs (customer service representatives) due to an affiliation effect, for example. It can also occur when any internal department assesses its own call center's calls (the Quality department, for example). To address this bias, organizations typically ask managers to evaluate calls from other departments than their own. Organizations may also secure an independent, third party to assess calls across multiple call centers within an organization. Using these approaches, organizations can often calibrate their familiar/internal assessments with those of unfamiliar/third part results. This calibration process enables assessors to be more consistent and accurate in assessing future calls. As indicated earlier in this paper, feedback on service delivery is rarely provided real time. Recordings of calls are necessary to provide a more timely way of providing feedback to an actual call in order to meet the second rule of feedback.

Once an assessment process is developed, the task becomes defining and measuring service delivery as a baseline. For inbound call centers, CSR skill and capability have been shown to directly affect the customer's experience and service quality as stated by rule 4. A study by Keiningham, Aksoy, Andreassen, Cooil and Wahren (2006) showed that service delivery can be defined by the dimensions of reliability, responsiveness, assurance and empathy - all attributes passed along by the CSR. Most call dimensions identified to improve service quality focus on the quality of communication, accuracy of the information and speed of the response/resolution. There have been studies in the literature that try to determine the most important dimension(s), and these studies have produced some mixed and situational results. However, there is no single list of dimensions that define customer satisfaction that fit all call centers. Clearly the goal should be to improve as many dimensions that a call center has determined that drive service quality and satisfaction for the greatest percentage of customers.

\section{Training on Dimensions}

To focus improvement on service delivery, rule 5 says that the dimensions of a good call should be identified and translated into CSR behaviors and skills. The dimensions in a CSR's ability to handle a call that has a positive impact on customer satisfaction do vary somewhat, but most can be consolidated into the following:

CSR communication that is: 1. courteous, friendly and attentive; 2. reliable and knowledgeable; 3.confident and helpful; and 4. prompt.

CSR data entry/collection that is: 1.accurate; and 2. relevant.

These definitions offer the first level definition of what service delivery is from the call center's perspective, and should be further defined prior to training CSRs. For example, courteous, friendly and attentive dimension can be further defined as:

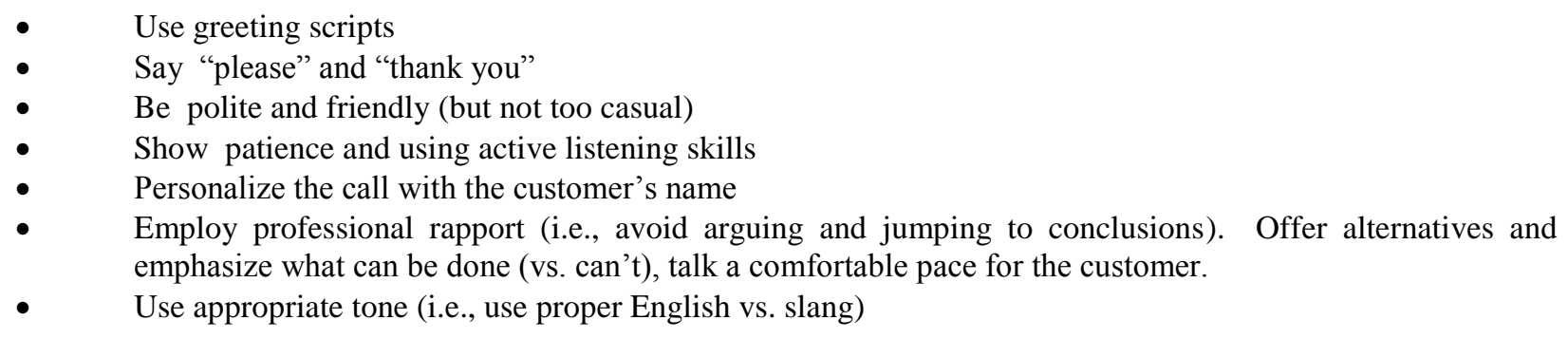

An effective CSR training roll-out should include the rationale for improving service delivery, a definition and explanation of the dimensions, several examples of actual calls that meet the dimensions and other information about how performance will be measured and/or rewarded.

The last rule of an effective feedback process implies management involvement, commitment and accountability where management owns the process. Manager training should include the same information as CSRs, and should also include additional information about the assessment process and how managers are expected 
to calibrate their assessments. In addition, some discussion around time management should also be included. For example, if a manager is assigned 10 CSRs and must review 6 calls per CSR per month, time must be allotted to listen to $60 \mathrm{CSR}$ calls per month, document the aspects of the call and provide this feedback to each CSR. These activities can represent a significant time investment for each manager, and care must be taken to position the assessment activities so that managers prioritize it appropriately. Their assessment efforts should also be measured each month to identify deviations as these occur. A common metric is to report the monthly percentage of assigned calls assessed (to include feedback provided to CSR) for each manager.

In terms of CSR training dimensions, some care must also be taken if a first call resolution dimension is employed. If this dimension is used to assess performance of CSRs, there are aspects of this dimension beyond the CSR's control. CSRs should be encouraged to be prompt by avoiding dead air and employing proper hold displays. They should also be encouraged to minimize any call delays that they control and take responsibility for the call (avoid rerouting a call that they can handle). But there are many aspects of first call resolution that are beyond the CSR's control. For example, long customer hold times are often a result of variations in other call center KSIs like forecasting, schedule adherence or service levels. Additional analysis and training often needs to be done to employ a first call resolution strategy effectively. For example, customer and/or call segments should be identified, routing configured and CSRs trained for transactions that will be required given the call routing protocol. Also, other KSIs (like average hold time) should be aligned to the first call resolution dimension prior to adding it as a service delivery dimension.

\section{Operations Linkages}

Once a service quality initiative is implemented, the process of continuous improvement will illustrate further linkages as shown in the operations block of the service profit chain (Figure 1). Training CSRs to handle routine calls requires a significant investment in communication, technology, products and procedures. Additional training for improving service delivery and/or initiating a first call resolution strategy will increase the time and efforts in training CSRs. If CSR turnover is high, it becomes harder to justify further investment into the development and training of CSRs. Call centers will be prompted to understand the reasons for CSR turnover and adjust their internal environments and/or recruiting processes accordingly. Call center management should also understand the links between any recruiting variables to those CSRs who are most effective in delivery high quality customer service within the current context of the call center environment.

\section{Global Considerations}

If call center operations have been outsourced, the improvement process described in this paper becomes much more complex and sophisticated. The increase in complexity is caused by the differences imposed by different languages and culture as the host country interacts with international customers. Geert Hofstede researched the impact of the national culture on an organization's culture, and developed a Cultural Dimension model with five dimensions to assess multicultural impact. These five dimensions are: Power Distance; Individualism; Masculinity; Uncertainty Avoidance; and Long-Term Orientation (Hofstede, 1980). Hofstede's model can be used by call centers that have been outsourced, and can lead to a greater understanding between different cultures to positively manage service delivery. The goal in using this model is to try to maximize the positives from different cultural perspectives and minimize the conflict that these differences can cause. More research needs to be done to further develop this model for countries that house call centers that serving international customers.

\section{CONCLUSION}

This paper validates the linkages in the service profit chain by illustrating the relationships between service quality, customer service and customer retention. It discusses how CSR capabilities can be used to positively impact customer service. It is recommended that any quality process used must measure customer satisfaction separately from service delivery, and any quality improvement process should follow the six rules for providing feedback on calls for process improvement. CSR training should focus on the why-what-how of improving service delivery, with specific examples and definitions available as examples. Manager training should emphasize the importance of 
consistent and calibrated assessment along with a time management plan to assess CSR calls. Improving service delivery should be viewed as an initiative that will allow the call center to work smarter, and not necessarily harder. Balancing call center KSIs with other business aspects (i.e., recruiting, performance reviews/rewards, etc.) will be encountered as service delivery is continuously improved.

\section{AUTHOR INFORMATION}

Elaine J. Labach. Shortly after graduating with a BS in Chemical Engineering, Elaine Labach began her career in the automotive industry. After receiving her MBA (University of Michigan) and PhD (Indiana University), Elaine held teaching, management and consulting positions, primarily in the service sector. She is currently affiliated with Troy University.

\section{REFERENCES}

1. Anonymous (1997). MCI's formula for quality customer service, International Journal of Retail \& Distribution Management, 25(3).

2. Bansal, H., \& Taylor, S. (1997). Investigating the relationship between service quality satisfaction and switching intentions. In Elizabeth, J. \& Joseph, C. (Eds.), Developments in marketing science, Academy of Marketing Science, Coral Gables, FL, pp. 304-313.

3. Crosby, P. (1979). Quality is free: The art of making quality certain, New York: Penguin Putnam.

4. Dabholkar, P., Shepherd, C., \& Thorpe, D. (2000). A comprehensive framework for service quality: An investigation of critical conceptual and measurement issues through a longitudinal study, Journal of Retailing, 18(2), pp. 139-73.

5. Gans, N., Koole, G., \& Mandelbaum, A. (2003). Telephone call centers: Tutorial, review, and research projects, Manufacturing and Service Operations Management, 5(2).

6. Heskett, J., Jones, T., Loveman, G., Sasser Jr., E., \& Schlesinger, L. (1994). Putting the service-profit chain to work. Harvard Business Review, March- April, 164-174.

7. Hofstede, G. (1980). Culture's consequences: International differences in work-related values. Beverly Hills, CA: Sage Publications.

8. Jaiswal, A. (2008). Customer satisfaction and service quality measurement in Indian call centres, Managing Service Quality, 18(4).

9. Keiningham, T., Aksoy, L., Andreassen, T., Cooil, B. and Wahren, B. (2006). Call center satisfaction and customer retention in a co-branded service context, Managing Service Quality, 16(3), 269-89.

10. Labach, E. (2009). Service operations curriculum recommendations for undergraduate business majors, American Journal of Business Education, 2(7).

11. Levin, G. (2007). Measuring the things that matter - a deep dive into seven key metrics that are most critical in gauging and securing customer satisfaction, loyalty and contact center effectiveness, Call Center Magazine, 20(3). 